\title{
Icon Use by Different Language Groups: Changes in Icon Perception in Accordance with Cue Utility
}

\author{
Siné McDougall ${ }^{1}$, Alexandra Forsythe ${ }^{2}$, and Lucy Stares ${ }^{1}$ \\ ${ }^{1}$ University of Wales Swansea, Swansea SA2 8PP \\ \{s.mcdougall, 1.a.stares\} @swansea.ac.uk \\ ${ }^{2}$ Queen's University Belfast, Belfast BH10 1TT \\ a. forsythe@qub.ac.uk
}

\begin{abstract}
This study shows that both icon and function label characteristics combine subtly to affect the way that individuals perceive icons. Icon users unconsciously use the cues available to them: the extent to which these cues are utilised depends on the language background of the user. In order to optimise design we therefore need know more, not only about the effects of function label characteristics, but also about how they merge with icon and user characteristics to affect usability.
\end{abstract}

\section{Introduction}

It has been assumed that icons offer a universal and international mode of communication [2] [5] and they are used in airports, stations, and other public spaces for this reason. However, although some icons appear to be universally recognised (see Figure 1(a)), others are more culturally specific (see Figure 1(b) [3]). Furthermore, when Smith \& Siringo [6] asked participants to match an icon with its function they found considerable differences between nationalities (India, Turkey, Singapore and the United States). Given that icons are typically presented along with their function written underneath to optimise comprehension [7], this study examines the extent to which different language groups are able to use function labels.

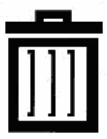

(a) Trashcan icon 'delete files'

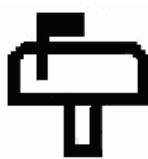

(b) North American mailbox 'electronic mail'
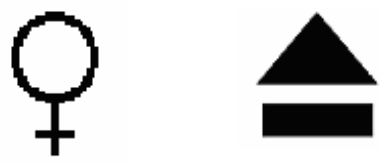

(c) 'female'

(d) 'eject'

Fig. 1. Examples of icons

Bates and her colleagues [1] suggest that language perception and comprehension depends on the information value of the cues available and the amount and type of 
processing associated with using each cue. We assumed that the same principles would hold true for icons and that icon perception would change depending on the utility of the cues available. The perceptions of two language groups were compared: one whose first language was English and another group from China who were acquiring English as their second language. Participants were asked to provide ratings of their subjective perceptions of the concreteness, or pictorialness, of icons when they were presented alone or when they were presented with the function label in English underneath. Our first hypothesis was that native English speakers' ratings would be more affected by the presentation of both icon and function since they would make more use of the function cue. Non-native English speakers were more likely to rate concreteness solely on the basis of pictorial cues in the icon than on concreteness of the function label since this cue would provide less information for them.

Other, more detailed, predictions were also made. Our second hypothesis was that the magnitude of the change in concreteness ratings for native English speakers would depend on the difference between the concreteness of the icon itself and the concreteness of the function label (i.e. on the combined use of both cues). Figure 1(c) and (d) illustrate how the icon and function label may, or may not, differ: 'female', a function rated as highly concrete, is depicted using an abstract icon while 'eject', an abstract function, is also depicted abstractly. For 'female', we might expect a large difference between 'icon only' and 'icon and function' concreteness ratings if English speakers are utilising both icons and function labels as cues, while differences between rating conditions would be minimal for 'eject'. A third hypothesis was that non-native English speakers would be more likely to use the function as a cue in their concreteness ratings if the words used in the function label occurred more frequently in English (i.e. were highly familiar). The assumption in both these hypotheses is that cue use is not all-or-none but is probabilistic, based on the combined information value of the cues available.

\section{Method}

\subsection{Participants}

A total of 99 participants took part in this study and all were student volunteers. Fifty-three participants rated icon concreteness when icons were presented alone or in combination with the function label. Twenty eight of these participants were attending English courses and their first language was Chinese; the remaining 25 participants were attending other courses and their first language was English. Each language group was divided into two further groups: 16 Chinese participants rated icons alone and 12 rated icons with function words; 12 English language speakers rated icons alone and 13 rated icons with function words. The English skills of both groups of non-native English speakers were equated using IELTS (International English Language Testing System) scores $(\mathrm{t}(26)=0.31, \mathrm{p}>0.05)$. Forty-six native English speakers provided additional ratings: 25 rated the concreteness of the function label alone and 21 participants rated their familiarity with the function label alone. 


\subsection{Materials and Procedure}

Participants were asked to rate 239 icons from a corpus used by McDougall et al [4] for which concreteness ratings had already been obtained. In contrast to the original ratings procedure for the corpus, where only icons without their functions were rated, two groups of participants were also presented with icons with the function label underneath. Ratings were on a 1-5 scale (5=definitely concrete, 1=definitely abstract)'. Icons were rated as concrete if they depicted real objects, materials or people; those that did not were rated as abstract. Additional ratings of the concreteness of the function label alone were also obtained using identical instructions. Ratings of familiarity with the function label were also obtained using a $1-5$ scale ( $1=$ very unfamiliar, $5=$ very familiar). The icons and ratings obtained can be seen at http://psy.swan.ac.uk/staff/mcdougall/additionalratings.htm.

\section{Results and Discussion}

In line with our first hypothesis, ratings for native English speakers differed between conditions $(\mathrm{M}$ (icon only $)=3.20, \mathrm{M}($ icon+function $)=3.02)$ while non-native speakers were remarkably similar irrespective of whether the function was shown underneath the icon or not $(\mathrm{M}$ (icon only $)=3.30, \mathrm{M}$ (icon+function $)=3.31)$. By-items analysis of variance with language (Chinese/English) and rating condition (icon only/ icon+function) as factors revealed a significant effect of language on ratings, $\mathrm{F}(1,238)=41.69, \mathrm{MSE}=9.12, \mathrm{p}<0.01$, since concreteness ratings were generally lower for English speakers. The effect of rating condition was also significant, $\mathrm{F}(1,238)=5.39$, $\mathrm{MSE}=2.12, \mathrm{p}<0.05$, as was the interaction between language and rating condition, $\mathrm{F}(1,238)=12.17$, $\mathrm{MSE}=1.76, \mathrm{p}<0.01$. This interaction shows that, as predicted, native speakers are more likely to change their perceptions of the icons given function cues than non-native speakers.

The second hypothesis stated that as the difference between icon and function concreteness increased (e.g. Figure 1(c)), so the difference in ratings obtained between the icon only and icon+function conditions would increase. This was examined by first computing the difference between the original norms (icons alone; see [4]) and ratings of the concreteness of the function name alone. This provided a measure of the difference between icon and function concreteness. The difference between ratings in the icon only and icon+function conditions was then calculated. Both of these difference scores were then correlated on the assumption that a significant correlation would occur if participants modified their ratings more given a greater difference between the concreteness of the icon and the concreteness of the function label. Correlations for both native and non-native speakers were significant $\left(r=.71\right.$ and $r=.43$ respectively). When Fisher's $z_{r}$ transformation was used to examine the difference between these two correlations, it revealed that the correlation for native speakers was significantly higher than for non-native speakers. This shows that subjective perceptions of icon concreteness are based on the difference in concreteness between icon and function for both groups but that native speakers use the function label cues more to adjust their perceptions of concreteness.

\footnotetext{
${ }^{1}$ Our thanks to Dr Chris Shei for translating ratings instructions for Chinese participants.
} 
Our final hypothesis was that Chinese speakers may be better able to use the cues provided by function labels if they were highly familiar. Icons whose function label familiarity ratings were in the top quartile were regarded as familiar $(n=59)$ and those in the bottom quartile were regarded as unfamiliar $(n=62)$. The same correlations were carried out as for Hypothesis 2 for non-native speakers for familiar and unfamiliar functions. Correlations for icons with familiar and unfamiliar functions were $\mathrm{r}=.58$ and .48 respectively. Fisher's $\mathrm{z}_{\mathrm{r}}$ transformations were used to compare these correlations with that for the whole icon set $(r=.43)$. While no differences were found between the whole sample and unfamiliar icons, the correlation was significantly higher when icons were familiar. This shows that second language English speakers are able to make better use of function names as cues when they occur more frequently.

\section{Conclusions}

The data obtained suggests that cross-linguistic differences in icon interpretation can be very subtle and affect the way icons are perceived. Even when not explicitly asked to do so, individuals use cues from icon and function in accordance with their ability to utilise those cues. At a practical level, designers need to know more about how icon and function characteristics act together to affect icon perception and interpretation and how the balance in the use of these cues changes with language and cultural background. Although our study showed that concreteness and familiarity are important cues, future research needs to examine the role of other cues such as the phonological and orthographic complexity of the function label and the closeness of the semantic relationship between icon and function.

\section{References}

1. Bates, E., Devescovi, A. \& Wulfeck, B.: Psycholinguistics: A cross-language perspective. Annual Review of Psychology, Vol. 52, (2001) 369-396

2. Bocker, M.: A multiple index approach for the evaluation of pictograms. Proceedings of the 14th International Symposium of Human Factors in Telecommunication (1993) 73-843.

3. Marcus, A.: Icon and symbol design issues for graphical user interfaces. In del Galdo, E.M., \& Nielsen, J. (eds.) International user interfaces, John Wiley \& Sons, New York (1996) 257-270

4. McDougall, S.J.P., Curry, M.B. \& de Bruijn, O.: Measuring symbol and icon characteristics: Norms for concreteness, complexity, meaningfulness, familiarity, and semantic distance for 239 symbols. Behavior Research Methods, Instruments \& Computers, Vol. 31. (1999) 487-519

5. Rogers, Y.: Icon design for the user interface. International Review of Ergonomics, Vol. 2. (1989) 129-154

6. Smith, J.A. \& Siringo, M.P. (1997). The acceptability of icons across countries. In G. Salvendy, M.J. Smith \& R.J. Koubek (eds.), Design of computing systems: Cognitive considerations: Advances in Human Factors/ Ergonomics Vol. 21A. Elsevier, New York (1997) 177-180

7. Weidenbeck, S.: The use of icons and labels in an end user application program: An empirical study of learning and retention. Behaviour \& Information Technology, Vol.18. (1999) 68-82 